\title{
Mini-Jets in Deep Inelastic Scattering at HERA
}

\author{
S. Osman * \\ Lund University, \\ Box 118, SE 221 00, Sweden, E-mail: sakar.osman@hep.lu.se
}

\begin{abstract}
The production of jets with low $P_{T}$, mini-jets, in deep inelastic electron-proton scattering is studied. Mini-jet multiplicities are presented as a function of the $P_{T}$ of the leading jet in bins of $\eta$ and $Q^{2}$. The analysis is performed for an inclusive jet sample, and for a dijet sample, where the second jet is required to have an azimuthal angle larger than 140 degrees with respect to the leading jet. The dijet sample is split into two samples which are enhanced in direct photon and resolved photon processes, respectively. The results are compared to various QCD based models. Here only the result for the inclusive jet sample will be presented.
\end{abstract}

\section{Introduction}

In electron-proton scattering the partonic content of the exchanged virtual photon may be resolved if the $P_{T}$ of the interacting partons is larger than $Q^{2}$ and thereby the photon will behave like a hadronic object. Thus, similar to hadron-hadron scattering there will be a certain probability that collisions between the resolved photon and the proton involve more than one parton interaction, multiple interactions (MI). Previous measurement in photoproduction at HERA [2] have shown that only models with MI give a satisfactory description of the data. This analysis constitutes the first study of possible MI in DIS from measurements of low $P_{T}$ jets produced in addition to the leading jet(s) of the event. The basic principle of the analysis is to define regions in phase space where contributions from the final state products originating from the primary hard interaction are expected to be small. The concept follows closely the one used by the CDF collaboration at the TEVATRON [3]

\section{Analysis Method}

The starting point is to define and isolate the leading jet(s) originated from the hard primary interaction and investigate the remaining regions for additional activities, which in this analysis comprise the presence of jets with low transverse momenta, mini-jets. Two different event samples are studied; inclusive jet events and dijet events, of which the latter constitutes a subsample of the inclusive sample. The analysis procedure is the following:

Inclusive jet sample: The leading jet is identified and reconstructed using the $k_{t}$-algorithm [4] in the h.c.m. rest frame. The jet with the highest transverse momentum in the h.c.m. rest frame is taken as the leading jet. The leading jet axis defines the azimuthal angle $\Delta \phi^{*}=0$ a. The region $\left|\Delta \phi^{*}\right|<60^{\circ}$ is defined as the 'toward region', and is expected to contain all particles belonging to the leading jet. The angular region $\left|\Delta \phi^{*}\right|>140^{\circ}$ is called the 'away region'. The transverse regions, $60^{\circ}<\left|\phi^{*}\right|<120^{\circ}$ are those where contributions from the primary collision should be small and the effects from additional activities should be most visible. Event by event a 'high activity'- and 'low activity' region are defined, depending on

\footnotetext{
* On behalf of the H1 Collaboration.

abservables in the h.c.m. frame are labeled with *
} 
which region contains the most and least transverse momentum, respectively. These four regions are shown. in Figure 2

Dijet sample: The dijet sample includes events having at least two jets, where the two reconstructed jets are required to be almost back-to-back. The leading jet is again defining $\Delta \phi^{*}=0$, whereas the jet axis of the jet with the second highest transverse momentum, the sub leading jet, is restricted to be inside the 'away region'. This leaves some angular space to accommodate the transverse spread of the jet within the 'away region'.

\section{Event Selection}

The analysis is based on data taken with the H1 detector in 1999/2000 using colliding positrons and protons at energies of $27.5 \mathrm{GeV}$ and 920 $\mathrm{GeV}$, respectively. The DIS events are selected by requiring a positron in the SPACAL calorimeter with $E_{e}^{\prime}$ $>9 \mathrm{GeV}, 156^{\circ}<\theta_{e}<175^{\circ}$ where $E_{e}^{\prime}$ and $\theta_{e}$ are the energy and polar angle of the scattered positron, respectively. The photon inelasticity, $y$, and the virtuality, $Q^{2}$, are determined using the electron method and must fulfill $0.1<y<0.7$ and $5<Q^{2}<100 \mathrm{GeV}^{2}$. The invariant mass of the hadronic final state, $\mathrm{W}$, is required to be higher than $200 \mathrm{GeV}$ in order to enhance small $x_{b j}$ contributions and to increase the

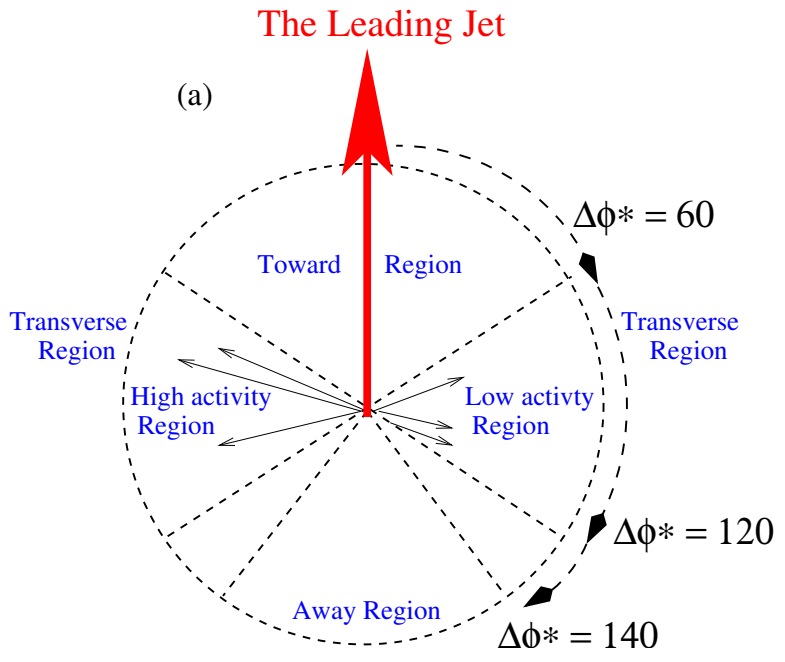

Figure 1: The transverse, toward and away regions. probability of mini-jet productions.

The inclusive jet sample consists of events that contain at least one jet, whereas the dijet sample includes events with at least two jets. For both samples the jet with the highest transverse momentum is chosen as the leading jet and in the dijet sample the sub leading jet has to fulfill the requirement $\left|\Delta \phi_{l s}^{*}\right|=\left|\phi_{l j}^{*}-\phi_{s j}^{*}\right|>140^{\circ}$, where $\phi^{*}$ is the jet azimuthal angle and the labels $l j$ and $s j$ denotes the leading and sub leading jets, respectively. The jets are reconstructed by the inclusive $k_{t}$-algorithm [4] in its $p_{t}$ weighting scheme mode, applied to combined object of tracks and calorimetric clusters in the h.c.m. rest frame. To ensure a good jet reconstruction it is required that the leading and sub leading jets must fulfill $-1.7<\eta^{l a b}<2.79$ and $P_{T}^{(*)}>5 \mathrm{GeV}$. Here, the pseudo-rapidity is given by $\eta_{j}=-\ln \left(\tan \left(\theta_{j} / 2\right)\right)$, where $\theta_{j}$ is the polar angle of the jet in the lab frame, and $P_{T}$ is the transverse energy of the jet. The pt cut are applied both in lab and h.c.m. rest frame.

Mini-jets are reconstructed with the same jet algorithm as the leading jets and within the same $\eta^{l a b}$ region. However, the minimum transverse momentum of a mini-jets is required to be larger than as $3 \mathrm{GeV}$ both in lab and h.c.m rest frame .

Data is corrected for limited detector resolution and acceptance using a bin-by-bin procedure. Correction factors are determined using detector simulated events, generated by 
the Monte Carlo programs RAPGAP [5] and DJANGO [6] with ARIADNE [7], where QED radiation has been taken into account.

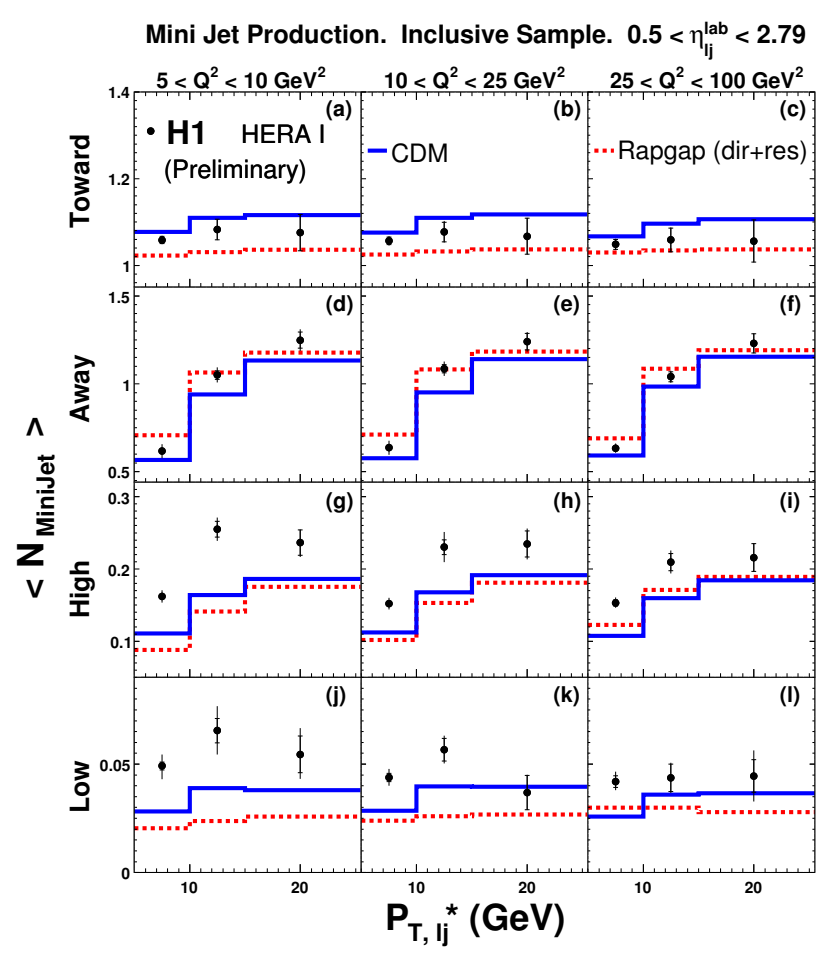

Figure 2: The average mini-jet multiplicity at the different $\Delta \phi^{*}$ regions in bins of $Q^{2}$ as a function of $P_{T}^{*}$ of the leading jet for the inclusive jet sample. The data is compared with the CDM model (solid line) and Rapgap (dashed line).

\section{Results}

The average multiplicity of mini-jets, $\left\langle N_{\text {minijet }}\right\rangle$, for the inclusive jet sample where the leading jet proceeds in the forward $\eta$ region are shown in Figures 2 - 3 for three bins of $Q^{2}$. The data are presented as a function of $P_{T}^{*}$ of the leading jet. Results have also been obtained for leading jets in the central region and for the dijet sample but they are not shown here, but can be found at [1]. The following can be observed:

The toward and away regions: All the different MC models, with or without MI, describe the 'toward region' well in all $Q^{2}$ bins, as expected. In the 'away region' there is an overall reasonable agreement for all models, Figures 2 - 3 (a-f).

The high activity region: The predictions of $\left\langle N_{\text {minijet }}>\right.$ by the MC models including no MI are generally too low in all $Q^{2}$-bins, Figure $2(\mathrm{~g}-\mathrm{i})$. PYTHIA + MI $[8,9]$ describes the data points fairly well in the lowest $Q^{2}$-bin and somewhat less well in the higher $Q^{2}$-bins, Figures 3 (g-i). 
The low activity region: The MC models with no MI included, significantly undershoots the data, Figure $2(\mathrm{j}-1)$, in all $Q^{2}$ bins. These deviations clearly increase with decreasing $Q^{2}$-values. PYTHIA + MI gives a much, Figure 3 (j-l), better description of data, although the deviations are still large in the highest $Q^{2}$-bin.

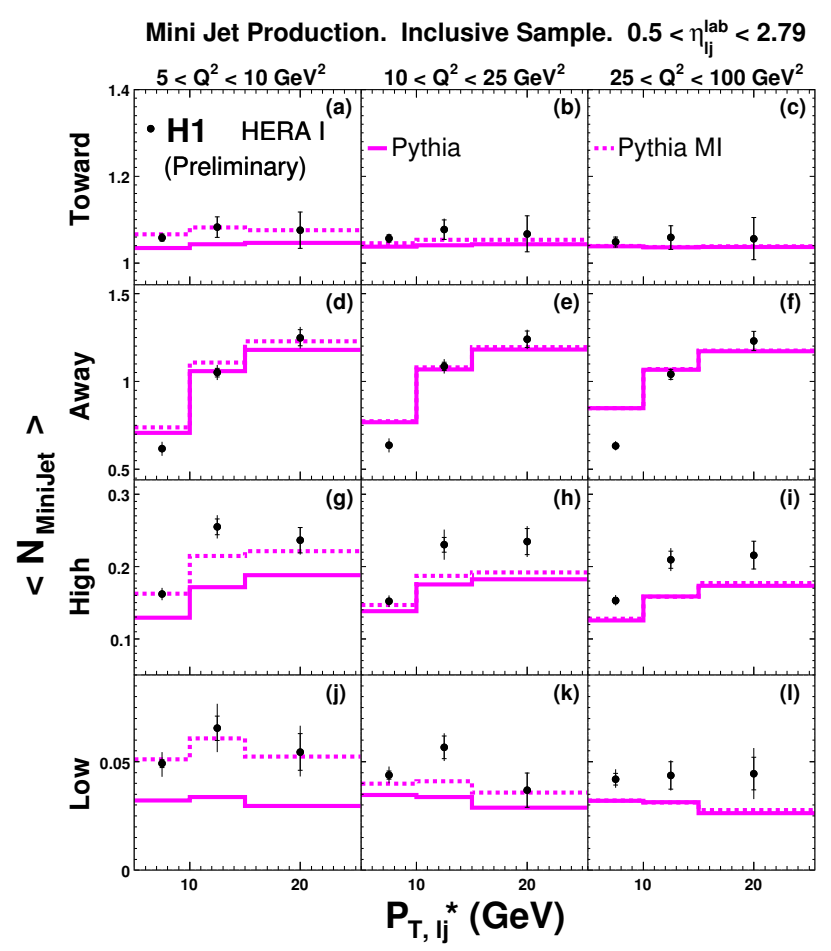

Figure 3: The average mini-jet multiplicity at the different $\Delta \phi^{*}$ regions in bins of $Q^{2}$ as a function of $P_{T}^{*}$ of the leading jet for the inclusive jet sample. The data is compared with Pythia (solid line) and Pythia with MI (dashed line).

\section{References}

[1] Slides:

http: //indico. cern. ch/contributionDisplay $\cdot$ py? contribId=226\&sessionId=6\&conf Id=9499

[2] S. Aid et al. [H1 Collaboration], Z. Phys. C70 (1996) 17.

[3] D. Acosta et al. [CDF Collaboration], Phys. Rev. D 65 (2002) 072005.

[4] S. D. Ellis and D. E. Soper, Phys. Rev. D 48(1993) 3160.

[5] H. Jung, Comput. Phys. Commun. 86 (1995) 147.

[6] K. Charchula, G. A. Schuler and H. Spiesberger, Comput. Phys. Commun. 81 (1994) 381.

[7] L. Lonnblad, Comput. Phys. Commun. 71 (1992) 15.

[8] T. Sjostrand, Phys. Lett. B 157 (1985) 321.

[9] T. Sjostrand, Comput. Phys. Commun. 82 (1994) 74. 